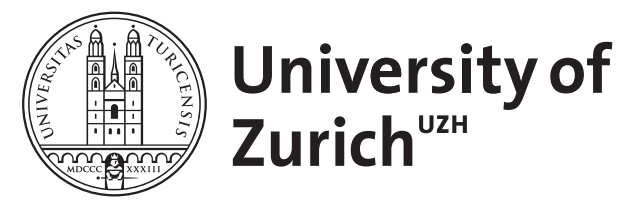

\title{
Palmitoylation and depalmitoylation defects
}

\author{
Hornemann, Thorsten
}

\begin{abstract}
Palmitoylation describes the enzymatic attachment of a 16-carbon atom fatty acid to a target protein. Such lipidation events occur in all eukaryotes and can be of reversible (S-palmitoylation) or irreversible (N-palmitoylation) nature. In particular S-palmitoylation is dynamically regulated by two opposing types of enzymes which add (palmitoyl acyltransferases - PAT) or remove (acyl protein thioesterases) palmitate from proteins. Protein palmitoylation is an important process that dynamically regulates the assembly and compartmentalization of many neuronal proteins at specific subcellular sites. Enzymes that regulate protein palmitoylation are critical for several biological processes. To date, eight palmitoylation related genes have been reported to be associated with human disease. This review intends to give an overview on the pathological changes which are associated with defects in the palmitoylation/depalmitoylation process.
\end{abstract}

DOI: https://doi.org/10.1007/s10545-014-9753-0

Posted at the Zurich Open Repository and Archive, University of Zurich

ZORA URL: https://doi.org/10.5167/uzh-105577

Journal Article

Published Version

Originally published at:

Hornemann, Thorsten (2015). Palmitoylation and depalmitoylation defects. Journal of Inherited Metabolic Disease, 38(1):179-186.

DOI: https://doi.org/10.1007/s10545-014-9753-0 


\title{
Palmitoylation and depalmitoylation defects
}

\author{
Thorsten Hornemann
}

Received: 22 May 2014 /Revised: 11 July 2014 / Accepted: 17 July 2014 / Published online: 5 August 2014

(C) SSIEM 2014

\begin{abstract}
Palmitoylation describes the enzymatic attachment of a 16-carbon atom fatty acid to a target protein. Such lipidation events occur in all eukaryotes and can be of reversible (S-palmitoylation) or irreversible (N-palmitoylation) nature. In particular S-palmitoylation is dynamically regulated by two opposing types of enzymes which add (palmitoyl acyltransferases - PAT) or remove (acyl protein thioesterases) palmitate from proteins. Protein palmitoylation is an important process that dynamically regulates the assembly and compartmentalization of many neuronal proteins at specific subcellular sites. Enzymes that regulate protein palmitoylation are critical for several biological processes. To date, eight palmitoylation related genes have been reported to be associated with human disease. This review intends to give an overview on the pathological changes which are associated with defects in the palmitoylation/depalmitoylation process.
\end{abstract}

\section{Protein palmitoylation and depalmitoylation}

The reversible enzymatic modification of proteins with a free fatty acid is generally described as S-acylation and occurs in all eukaryotes. It is different from other cellular lipidation

Communicated by: Matthias Baumgartner

Presented at the workshop "Diagnostic Approach, and Classification of IEM Affecting the Synthesis and Catabolism of Complex Lipids" in Paris, France, June 14-15, 2013.

T. Hornemann $(\bowtie)$

Institute for Clinical Chemistry, University Hospital Zurich, Raemistrasse 100, CH-8091 Zurich, Switzerland

e-mail: thorsten.hornemann@usz.ch

T. Hornemann

Institute of Physiology and Zurich Center for Integrative Human Physiology (ZIHP), University of Zurich, CH- 8057 Zurich, Switzerland events such as farnesylation and isoprenylation in which the lipid moiety is irreversibly attached to proteins via an amide or thioether bond. The most common modification is Spalmitoylation which describes the reversible attachment of a palmitic acid onto a cysteine residue via thioester linkage (Resh 2006; Conibear and Davis 2010; Salaun et al 2010). A diverse group of proteins undergo palmitoylation including signaling proteins, ion channels, scaffold proteins, proteins involved in vesicle trafficking and viral proteins (Mitchell Vasudevan et al.et al 2006; Charollais and Van Der Goot 2009; Fukata and Fukata 2010; Veit et al 2013). Up to date there is no consensus motive established which dictates Spalmitoylation. The attached palmitate serves as a hydrophobic anchor for proteins that lack transmembrane domains. The hydrophobicity of a single fatty acid alone is typically not sufficient to stably anchor a protein to a lipid bilayer. Therefore a second signal is normally required for stable membrane binding. Normally, this second signal is either a cluster of positively charged amino acids (lysines and arginines) or an attached palmitate (El-Husseini et al 2000; Fukata and Fukata 2010). Palmitoylation of a protein is typically associated with its transport to specific intracellular compartments. Soluble proteins are normally palmitoylated at the Golgi from where they traffic to the plasma membrane (Rocks et al 2010). The reversible nature of palmitoylation allows proteins to dynamically shuttle between intracellular compartments or to relocalize in physiological contexts. The small GTPases HRAS and NRAS shuttle between Golgi and plasma membrane due to a palmitoylation-depalmitoylation cycle (Rocks et al 2006). The dynamic nature of protein palmitoylation/depalmitoylation cycles resembles hereby the principle of regulating protein functions by a kinase and phosphatase mediated addition and removal of phosphate groups in cellular signaling events. Besides membrane interaction, palmitoylation also regulates protein stability, protein sorting, and the localization to specific membrane sub- 
domains (Linder and Deschenes 2007; Greaves et al 2009; Fukata and Fukata 2010). The segregation of plasma membrane proteins into lipid rafts is important for regulating many cell signaling events and often requires palmitoylation (Brown 2006; Levental et al 2010). Protein palmitoylation is important for the assembly and compartmentalization of many neuronal proteins at specific subcellular domains (Prescott et al 2009; Fukata and Fukata 2010) and plays a critical role in synaptic plasticity (El-Husseini and Bredt 2002; Huang and ElHusseini 2005). It is also important for neuronal developmental processes, such as neurite outgrowth, axon pathfinding, filopodia formation, and spine development (Kutzleb et al 1998; Kato et al 2000; Laux et al 2000; Gauthier-Campbell et al 2004; Arstikaitis et al 2008). It has been reported for numerous neuronal proteins, including signaling proteins (HRAS, NRAS and rhoB), synaptic scaffolding proteins (PSD95, GRIP1 and AKAP18), transmembrane proteins (G protein-coupled receptors), neuronal cell adhesion molecules (NCAMs), glutamate receptors (GluRs), synaptic vesicle proteins (SNAP25), and cysteine string proteins (CSP and synaptotagmin I) (El-Husseini and Bredt 2002; Linder and Deschenes 2007). In particular the postsynaptic targeting of PSD-95, a molecule involved in excitatory synapse development and plasticity, is regulated by palmitoylation (ElHusseini et al 2000; Kim and Sheng 2004). Glutamate receptor activation markedly accelerates depalmitoylation of PSD95 and causes endocytosis of the associated AMPA ( $\alpha$ amino-3-hydroxy-5-methyl-4-isoxazole propionic acid) glutamate receptor (AMPAR) which leads to a down regulation of the respective signaling pathway (El-Husseini et al 2002). Palmitoylation can also be influenced by alternative splicing. This is well described for Cdc42, a small Rho GTPase that is involved in neuronal morphogenesis. Cdc41 is normally prenylated but brain specific alternative splicing results in a variant that becomes palmitoylated instead. Both variants are expressed in developing neurons but only the palmitoylated isoform induces the extension of dendritic filopodia which then develop into dendritic spines (Kang et al 2008).

Instead of forming a thioester bond acylation may also occur at an $\mathrm{N}$-terminal cysteine or glycine via an amide bond (N-palmitoylation). N-palmitoylation is primarily found in secreted proteins (Nadolski and Linder 2007) and is like prenylation and myristoylation not reversible and therefore not dynamic.

\section{Palmitoyl acyltransferases (PATs) and thioesterases}

The lipidation of proteins was first observed 30 years ago (Schmidt and Schlesinger 1979; Schlesinger et al 1980) but only recently the relevant catalytic enzymes were identified. The first palmitoyl acyltransferase was identified in $S$. cerevisiae by forward genetic screens. This approach identified Erf2-Erf4 (Bartels et al 1999; Lobo et al 2002 and Akr1
(Roth et al 2002) as palmitoyl acyltransferases (PATs) for the yeast proteins RAS2 and Yck2 (casein kinase 2). Erf2 and Akr1 share a 51-amino acid domain known as DHHC cysteine-rich domain (CRD) which is a variant of the $\mathrm{C}_{2} \mathrm{H}_{2}$ zinc finger motif (Putilina et al 1999). Erf2 and Akr1 strictly required an intact DHHC motif for activity and subsequent work showed that palmitoyl transferases are general members of the DHHC protein family (although commonly referred to as "DHHC PATs" the official gene name is "ZDHHC", e.g., ZDHHC 8 ). The core DHHC motif is highly conserved and essential for catalytic function (Mitchell et al 2006). Seven DHHC genes have been identified in yeast and 23 DHHC proteins are predicted from mammalian genomes (human and mouse) (Fukata, Fukata et al 2004; Ohno et al 2006). DHHC palmitoyl transferases are membrane proteins with four to six predicted transmembrane domains (TMD) (Politis et al 2005; Conibear and Davis 2010) and expression studies showed that the majority of the mammalian PATs are localized at the ER and Golgi (Ohno et al 2006) with some exceptions like DHHC5, which is localized to the plasma membrane. Erf2 is present in the endoplasmic reticulum (ER), whereas Akr1 is localized at the Golgi. A precise consensus sequence for palmitoylation has not been identified yet and it seems that some DHHC proteins can palmitoylate rather a broad range of substrates whereas others are more selective. Also Erf2 and Akr1 showed a certain substrate specificity as Yck2 palmitoylation is unaffected by mutation in Erf2 and Ras2 palmitoylation is not affected by $A k r 1$ mutants. However, the lack of a consensus sequence currently does not allow to predict whether a protein is a target for palmitoylation (Fig. 1).

In contrast to S-palmitoylation is N-palmitoylation mediated by set of multispanning transmembrane $\mathrm{O}$-acyltransferase (MBOAT). Many members of the MBOAT family are lysophospholipid acyltransferases (Shindou and Shimizu 2009) and typically catalyze the addition of a fatty acid to the hydroxyl group of membrane lipids (Hofmann 2000).

In contrast to the great number of DHHC PATs only four acyl protein thioesterases (commonly referred to as thioesterases) have been identified to catalyze depalmitoylation. This is APT1 (LYPLA1) and APT2 (LYPLA2), and the protein palmitoyl thioesterases 1 (PPT1) and 2 (PPT2) (Zeidman et al 2009; Tomatis et al 2010). APT1 was first purified from the cytosol of rat hepatocytes based on its ability to remove palmitate from $\left[{ }^{3} \mathrm{H}\right]$-palmitoyl- $\mathrm{G} \alpha_{\mathrm{i}}$ (Duncan and Gilman 1998). APT1 is expressed in a wide range of mouse tissues and has been shown to depalmitoylate a list of proteins including H-Ras (Duncan and Gilman 1998) and endothelial NOS (Yeh et al 1999). APT2 is a homologue of APT1 and shows $64 \%$ amino acid sequence identity. It has been shown to depalmitoylate GAP-43 and also H-Ras (Tomatis et al 2010; Rusch et al 2011). Also thioesterases seem not to have a defined substrate recognition sequence and the list of APT1 substrates contains proteins that are 
Fig. 1 Schematic representation of the interplay between palmitoyltransferases (PAT, blue) and acyl protein thioesterases (orange) and associated pathologies

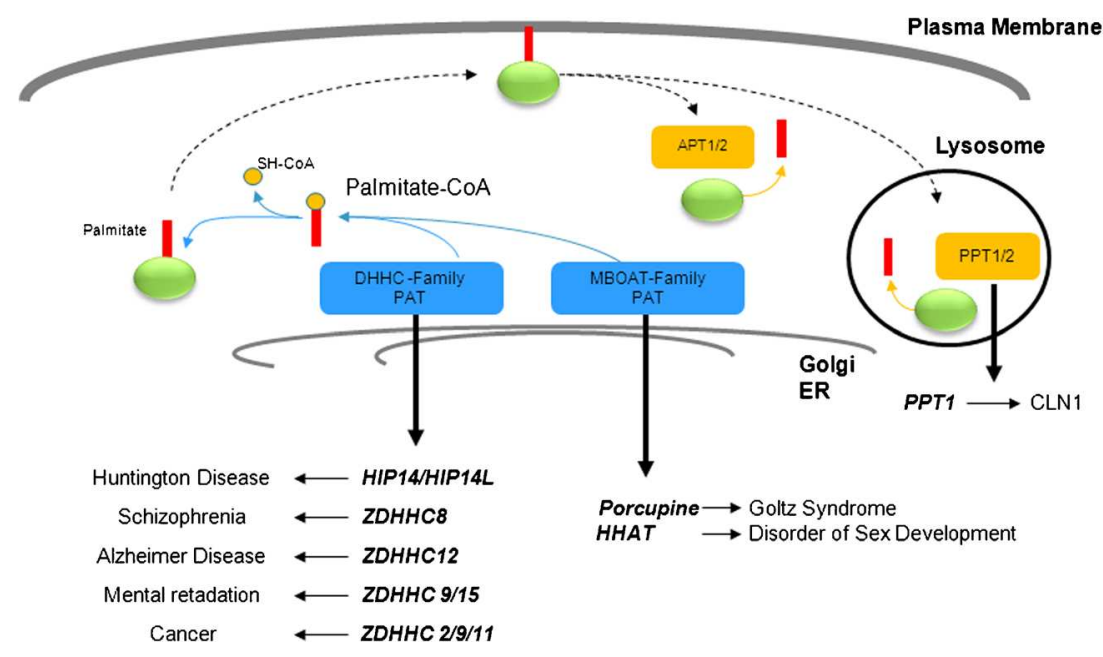

structurally diverse and containing different lipid modifications (Zeidman et al 2009). The third identified thioesterase is PPT1, which is a lysosomal enzyme and indiscriminately cleaves fatty acids from cysteine residues in the process of protein degradation (Zeidman et al 2009). As PPT1 is located in the lysosomes it is probably not involved in the dynamic depalmitoylation of cytoplasmic proteins (Hellsten et al 1996; Verkruyse and Hofmann 1996). However, PPT1 interacts with the $\mathrm{F}(1)$-complex of the mitochondrial ATP synthase and the levels of $F(1)$-subunits alpha and beta on the plasma membrane were increased in neurons of PPT1 deficient mice (Lyly et al 2008). PPT2 is a homologue to PPT1 and shares about $20 \%$ identity. Also PPT2 is a lysosomal protein but seems to have a distinct substrate specificity as it did not remove palmitate groups from proteins that are substrates for PPT1 (Soyombo and Hofmann 1997).

Enzymes that regulate protein palmitoylation are critical for several biological processes. To date, nine palmitoylation related genes have been reported to be associated with human disease (Liu et al 2002; Mansouri et al 2005; Yanai et al 2006; Raymond et al 2007; Mizumaru et al 2009; Singaraja et al 2011; Callier et al (2014).

\section{Huntington's disease}

Huntington's disease (HD) is a neurodegenerative disease that presents with cognitive, motor, and psychiatric signs and symptoms (Roos 2010; Sturrock and Leavitt 2010). The prevalence of HD in Caucasian European populations is 5-7 per 100,000 individuals (Warby et al 2011). HD is caused by mutations in the HTT (huntingtin or HD) gene located on chromosome 4. The exact function of HTT is not clear. It is essential for development and the absence of HTT is lethal in mice (Nasir et al 1995). The protein has no sequence homology to other proteins and is highly expressed in neurons and testes of humans and rodents (Cattaneo et al 2005). CAG repeats which exceed the number of 36 lead to the production of an HTT protein with an abnormally long N-terminal polyglutamine tail which is unstable. Individuals with 36 to 40 CAG repeats may or may not develop HD whereas individuals with more than 40 repeats will develop the disorder. Sixty and more CAG repeats lead to a severe form of juvenile HD.

Huntingtin has been found to interact with a number of other proteins including HIP14 (ZDHHC17) (Kalchman et al 1996; Singaraja et al 2002). HIP14 and its homologue HIP14L are human orthologues of the yeast proteins Akr1 and Akr2. HIP14L is an atypical ZDHHC protein as it has a DQHC instead of the typical DHHC motive (Mitchell et al 2006; Greaves and Chamberlain 2011). The role of HIP14 in HD gained focus when it was shown that the HIP14-HTT interaction correlated inversely with the number of CAG repeats. This suggests an aberrant palmitoylation to be involved in the pathogenesis of HD (Singaraja et al 2002). HIP14 is expressed in brain and in particular in medium spiny neuron (MSN) cells, special inhibitory neurons which are primarily present in corpus striatum of the basal ganglia. MSN cells play a key role in initiating and controlling movements of the body, limbs, and eyes and are the earliest cell population affected in HD. Further work showed that HTT is primarily palmitoylated at Cys214 and the mutation of this site was associated with increased inclusion formation in COS cells and neuronal cultures and increased NMDA-induced toxicity in cultured rat cortical neurons (Yanai et al 2006). The silencing of HIP14 in cortical neuron cultures of YAC128 mice, a mouse model which is transgenic for the human huntingtin protein, resulted in increased inclusion formation (Slow et al 2003; Yanai et al 2006). Palmitoylation of HIP14 is reduced in brains of YAC128 mice, suggesting a reduced HIP14 activity in the presence of mutant HTT. The overexpression of HIP14 reduced inclusion formation in cultured neurons (Yanai et al 2006). HIP14 itself is autopalmitoylated (Huang et al 2004) a conserved feature that is correlated with PAT activity (Fukata, Fukata et al 2004; Huang et al 2004). 
Autopalmitoylation is reduced in HIP14 isolated from YAC128 brains. HIP14 appears to be predominantly a neuronal PAT and its confirmed substrates include SNAP25, GAD65, HTT, STREX-BK potassium channel, and GluR1/2 AMPA receptor subunits (Fukata, Fukata et al 2004; Huang et al 2004; Huang et al 2009; Greaves et al 2010; Tian et al 2010; Singaraja et al 2011). HIP14 may also be a PAT for PSD-95 as the knockdown of HIP14 reduced palmitoylation of PSD-95 (Huang et al 2004, 2009). In return it appears that HTT modulates the palmitoylation and activity of HIP14 itself. Palmitoylation of HIP14 was reduced in heterozygous $\mathrm{Hdh}^{+/-}$mice. Hip $14^{-/-}$mice revealed a reduced brain weight at 1 month of age (Singaraja et al 2011).

\section{Schizophrenia}

For three SNPs in the palmitoyl acyltransferases $Z D H H C 8$ a significant association with schizophrenia was found in a US and South African population (Liu et al 2002). The association with schizophrenia was further confirmed for one of these SNPs (rs175174) in an American and South African cohort. An association between $\mathrm{ZDHHC} 8$ and schizophrenia was also found in the Han Chinese population (Chen et al 2004b). Also the association of the 22q11 microdeletion region, which bears the ZDHHC8 gene and schizophrenia was reported. The identified SNP was functional and influenced alternative splicing of $Z D H H C 8$ which led to the retention of intron- 4 and the introduction of a premature termination codon. Female ZDHHC8-deficient mice showed abnormalities in fear-related measures of spontaneous activity which were absent in male mice. This was believed to be related to an influence of ZDHHC8 on glutamatergic signal transmission, as female ZDHHC8-deficient mice also appeared to be less sensitive to an NMDAR blocker (Mukai et al 2004). A follow-up study demonstrated that $Z D H H C 8$-deficient mice have a decreased density of dendritic spines (Mukai et al 2008). Furthermore it was shown that a polymorphism in ZDHHC8 is associated with nystagmus (abnormalities in smooth eye movements) which is common in schizophrenia (Shin et al 2010). However, several other studies failed to identify an association of ZDHHC8 with schizophrenia in other populations (Glaser et al 2005, 2006; Otani et al 2005; Saito et al 2005; Demily et al 2007; Xu et al 2010).

\section{Alzheimer's disease}

A number of studies have explored the role of palmitoylation in the pathogenesis of Alzheimer's disease (AD). Alzheimer's disease is a neurodegenerative type of dementia in which the death of brain cells causes memory loss and cognitive decline. The disease starts typically mild and gets progressively worse. A typical pathogenic step in $\mathrm{AD}$ is the generation of neurotoxic beta-amyloid $(A \beta)$ from amyloid precursor protein
(APP) by the sequential cleavage of two proteases ( $\beta$ - and $\gamma$-secretase). No genetic link has yet been found between PATs and AD but there is evidence that the $\beta$ - and $\gamma$ secretase enzymes are palmitoylated. Whether this palmitoylation is altered in $\mathrm{AD}$ is currently unclear. ZDHHC12 has been linked to APP trafficking by retaining APP in the Golgi and to prevent its further trafficking to the trans Golgi network and PM in neuroblastoma cells (Mizumaru et al 2009). However, this is probably not a direct effect of APP palmitoylation by ZDHHC12 as APP does not contain cytosolic cysteines.

Interestingly the major APP cleaving enzyme BACE1 is palmitoylated at four sites: three within the C-terminal cytosolic tail (Benjannet et al 2001) and one on its TMD which seems to be critical for targeting BACE1 to lipid rafts. Five PATs (ZDHHC3, 4, 7, 15, and 20) have been identified by coexpression studies to potentially enhance the palmitoylation of BACE1 (Vetrivel et al 2009).

\section{Goltz syndrome}

Human focal dermal hypoplasia or Goltz syndrome is an Xlinked dominant form of ectodermal dysplasia. It is transmitted as an X-linked dominant trait and is lethal in utero for male fetuses. It is a multisystem disorder, primarily characterized by skin manifestations as atrophic and hypoplastic areas (Wang et al 2007). Clinical signs constitute areas of cutaneous atrophy and periorificial papillomas around the mouth, genitalia, and/or anus. Osseous defects include scoliosis, hypoplastic clavicles and ribs, and a deformed thorax. Dental anomalies are typical and may include malpositioned teeth, extra teeth, and enamel defects. Eyes are affected by coloboma of the iris, microphthalmia, and/or strabismus. Goltz syndrome is caused by mutations in the Porcupine (Porcn) gene. Porcn is a multipass membrane protein and a member of the MBOAT family. Porcupine (Porcn) has been implicated in fatty acid modifications of Wnt proteins (Willert et al 2003; Takada et al 2006; Galli et al 2007) which are dual acylated with palmitate and palmitoleate. This modification is required for Wnt protein secretion and signaling (Takada et al 2006; Doubravska et al 2011) and dysregulation of the Wnt signaling pathways is associated with oncogenesis (Polakis 2007). The deletion of Porcn in mice is embryonically lethal due to the failure to secrete functional Wnt proteins (Biechele et al 2011) but symptoms of Goltz syndrome can be recapitulated in mice with a conditional disruption of Porcn (Barrott et al 2011).

Other diseases related to PAT mutations

The post-translational attachment of cholesterol and palmitate to the Hedgehog $(\mathrm{Hh})$ family of secreted proteins is critical for multimerization and long range signaling potency (Chen et al 2004a). Hh proteins act as morphogens to control embryonic 
patterning and development in a variety of organ systems. A very recent study reported the case of an autosomal recessive syndromic 46,XY disorder of sex development (DSD) with testicular dysgenesis and chondrodysplasia which was associated with a homozygous missense mutation in the hedgehog acyl-transferase (HHAT) gene (Callier et al 2014). The mutation $(\mathrm{G} 287 \mathrm{~V})$ was found in a conserved membrane bound Oacyltransferase (MBOAT) domain and in vitro studies showed that the mutations disrupted the ability of HHAT to palmitoylate $\mathrm{Hh}$ proteins such as $\mathrm{DHH}$ and $\mathrm{SHH}$.

A translocation between chromosome $\mathrm{X}$ and a region 15,2442 bp upstream of the ZDHHC15 gene which resulted in the absence of ZDHHC15 transcripts was reported in the case of a 29-year old woman with severe non-syndromic mental retardation (Mansouri et al 2005). Mutations in another X-chomosomal PAT (ZDHHC9) were found in four of 250 families with $\mathrm{X}$-linked mental retardation. Two missense mutations were located in the DHHC-CR domain and one was located at a highly conserved residue (Mitchell et al 2006; Raymond et al 2007). However, the effect of these mutations on enzyme activity was not yet validated in functional assays.

Mutations in three PATs (ZDHHC2, 9, 11) have been implicated in various forms of human cancer (Oyama et al 2000; Mansilla et al 2007; Yamamoto et al 2007; Zhang et al 2008) and one study reported HIP14 to be an oncogene in vitro and in vivo in mice (Ducker et al 2004). Mutations in HIP14L (ZDHHC13) and ZDHHC21 have been shown to result in dermatological and related phenotypes in inbred mice (Mill et al 2009; Saleem et al 2010).

\section{Ceroid lipofuscinosis type $1(C L N 1)$}

Besides defects in the palmitoylation reaction is also the opposite step - the removal of the S-linked palmitate associated with pathological conditions. Mutations in the PPT1 gene result in infantile neuronal ceroid lipofuscinosis type 1 (CLN1 or Batten disease) (Vesa et al 1995). CLN1 is a rare disease (prevalence one out of 100,000 births) and belongs to the family of neuronal ceroid lipofuscinosis (NCL) a genetically distinct group of neurodegenerative diseases featured by epilepsy, progressive blindness and premature death. Collectively, they represent the most common group of hereditary encephalopathies in childhood, with an incidence of up to $1 / 12,500$ (Haltia 2006). At the ultrastructural level the disease is associated with the lysosomal accumulation of lipofuscin - a granular autofluorescent lipopigment. CLN1 is caused by homozygous or compound heterozygous mutations in the PPT1 gene on chromosome 1p32. To date 64 PPT mutations are annotated in the NCL mutation database (http:// www.ucl.ac.uk/ncl/mutation.shtml). Many of these mutations are private mutations although a c.451 C $>$ T (R151X) exchange seems to be the most prevalent mutation in nonrelated carriers. A c.364 A $>$ T (R122W) transversion seems to cluster in Finnish patients. Both mutations represent about $20 \%$ of the total CLN1 cases. CLN1 has an early-onset and symptoms appear typically within the first 6-12 months of life with previously normal development (Mitchison et al 1998). The disease is initially characterized by the delay of developmental progress, microcephaly, and the loss of motor function leading to hypotonia. Vision loss becomes apparent from the 12th month and progresses to blindness until 2 years of age. At that time the child usually starts to loose previously acquired skills (speech and movements) and most affected children die by the age of seven (Santavuori 2011). The autopsy shows a shrunken brain and diffuse cortical and cerebellar atrophy. Electron microscopy of brain and other tissues like blood lymphocytes demonstrates accumulation of granular osmiophilic dense (GROD) bodies. Palmitoylated peptide intermediates accumulate in the lysosomes along with sphingolipid activator proteins (saposin) A and D (Tyynela et al 1993). After the crystal structure of PPT1 was identified, a correlation was observed between the severity of the infantile CLN1 phenotype and the effect of the various mutations on the catalytic site (Bellizzi et al 2000). The pathogenesis of the disease may be due to activation of an apoptosis pathway. PPT1 deficient mice demonstrate abnormal ER morphology and an accumulation of palmitoylated GAP-43 in the ER. This might lead to activation of unfolded protein response in the ER and subsequently activation of caspase-3 and apoptosis (Zhang et al 2006). The same group reported that caspase-9 is activated following increased production of reactive oxygen species and disruption of calcium homeostasis. PPT1 may therefore help to protect against apoptosis. Neuroblastoma cells which overexpress PPT1 showed reduced cell death, reduced activation of caspase-3, and increased phosphorylation of the anti-apoptotic protein Akt when treated with the apoptosis-inducing agent $\mathrm{C}_{2}$ ceramide (Cho and Dawson 2000). On the other hand, inhibition of PPT1 either via PPT1 antisense RNA or a PPT1 inhibitor resulted in enhanced apoptosis (Cho et al 2000).

Experimental PPT2 deficiency in mice causes an unusual form of neuronal ceroid lipofuscinosis with striking visceral manifestations but no PPT2 deficiency has been described in humans yet (Gupta et al 2003).

Currently there is no treatment for CNL1. However, a recently concluded pilot study investigated the combination of phosphocysteamine and $\mathrm{N}$-acetylcysteine as a therapy in nine patients with CNL1 (Levin et al 2014). The results showed a remarkable clearance of GRODs in blood cells, a reduced irritability of the patients and a slowdown of the disease course. In particular the reduced irritability in response to the treatment might affect present clinical practice. Besides these promising results seizures may be controlled or reduced with use of anti-epileptic drugs and physical, speech, and occupational therapies may help affected patients retain their cognitive and motor functions as long as possible. 
In summary six PATs (HIP14, HIP14L, ZDHHC8, ZDHHC9, ZDHHC12, and ZDHHC15) which catalyze Spalmitoylation have been implicated in the neuropsychiatric diseases like Alzheimer's and Huntington's disease, schizophrenia, and mental retardation. Mutations in Porcupine and HHAT which both belong to the MBOAT family cause human focal dermal hypoplasia (Goltz syndrome) and testicular dysgenesis and chondrodysplasia, respectively. On the opposite side are defects in the thioesterase PPT1 associated with infantile NCL (CLN1). This highlights the importance of protein palmitoylation in particular for neurons as a disturbance of this process results in severe, mostly neuronal pathologies.

Acknowledgments T. Hornemann is grateful to the Gebert Rüf Foundation (GRS-047/09), the Center of Integrated Human Physiology (ZIHP) and "radiz" - Rare Disease Initiative Zurich, Clinical Research Priority Program for Rare Diseases, University of Zurich.

Compliance with ethics guidelines This article does not contain any studies with human or animal subjects performed by the author

Conflict of interest None.

\section{References}

Arstikaitis P, Gauthier-Campbell C, Carolina Gutierrez Herrera R et al (2008) Paralemmin-1, a modulator of filopodia induction is required for spine maturation. Mol Biol Cell 19(5):2026-2038

Barrott JJ, Cash GM, Smith AP, Barrow JR, Murtaugh LC (2011) Deletion of mouse Porcn blocks Wnt ligand secretion and reveals an ectodermal etiology of human focal dermal hypoplasia/Goltz syndrome. Proc Natl Acad Sci U S A 108(31):12752-12757

Bartels DJ, Mitchell DA, Dong X, Deschenes RJ (1999) Erf2, a novel gene product that affects the localization and palmitoylation of Ras2 in Saccharomyces cerevisiae. Mol Cell Biol 19(10):6775-6787

Bellizzi JJ 3rd, Widom J, Kemp C et al (2000) The crystal structure of palmitoyl protein thioesterase 1 and the molecular basis of infantile neuronal ceroid lipofuscinosis. Proc Natl Acad Sci U S A 97(9): 4573-4578

Benjannet S, Elagoz A, Wickham L et al (2001) Post-translational processing of beta-secretase (beta-amyloid-converting enzyme) and its ectodomain shedding. The pro- and transmembrane/cytosolic domains affect its cellular activity and amyloid-beta production. J Biol Chem 276(14):10879-10887

Biechele S, Cox BJ, Rossant J (2011) Porcupine homolog is required for canonical Wnt signaling and gastrulation in mouse embryos. Dev Biol 355(2):275-285

Brown DA (2006) Lipid rafts, detergent-resistant membranes, and raft targeting signals. Physiology 21:430-439

Callier P, Calvel P, Matevossian A et al (2014) Loss of function mutation in the palmitoyl-transferase HHAT leads to syndromic 46, XY disorder of sex development by impeding Hedgehog protein palmitoylation and signaling. PLoS Genet 10(5): e1004340

Cattaneo E, Zuccato C, Tartari M (2005) Normal huntingtin function: an alternative approach to Huntington's disease. Nat Rev Neurosci 6(12):919-930
Charollais J, Van Der Goot FG (2009) Palmitoylation of membrane proteins (Review). Mol Membr Biol 26(1):55-66

Chen MH, Li YJ, Kawakami T, Xu SM, Chuang PT (2004a) Palmitoylation is required for the production of a soluble multimeric Hedgehog protein complex and long-range signaling in vertebrates. Genes Dev 18(6):641-659

Chen WY, Shi YY, Zheng YL et al (2004b) Case-control study and transmission disequilibrium test provide consistent evidence for association between schizophrenia and genetic variation in the 22q11 gene ZDHHC8. Hum Mol Genet 13(23):2991-2995

Cho S, Dawson G (2000) Palmitoyl protein thioesterase 1 protects against apoptosis mediated by Ras-Akt-caspase pathway in neuroblastoma cells. J Neurochem 74(4):1478-1488

Cho S, Dawson PE, Dawson G (2000) Antisense palmitoyl protein thioesterase 1 (PPT1) treatment inhibits PPT1 activity and increases cell death in LA-N-5 neuroblastoma cells. J Neurosci Res 62(2): 234-240

Conibear E, Davis NG (2010) Palmitoylation and depalmitoylation dynamics at a glance. J Cell Sci 123(Pt 23):4007-4010

Demily C, Legallic S, Bou J et al (2007) ZDHHC8 single nucleotide polymorphism rs175174 is not associated with psychiatric features of the 22q11 deletion syndrome or schizophrenia. Psychiatr Genet 17(5):311-312

Doubravska L, Krausova M, Gradl D et al (2011) Fatty acid modification of Wnt1 and Wnt3a at serine is prerequisite for lipidation at cysteine and is essential for Wnt signalling. Cell Signal 23(5):837-848

Ducker CE, Stettler EM, French KJ, Upson JJ, Smith CD (2004) Huntingtin interacting protein 14 is an oncogenic human protein: palmitoyl acyltransferase. Oncogene 23(57):9230-9237

Duncan JA, Gilman AG (1998) A cytoplasmic acyl-protein thioesterase that removes palmitate from $G$ protein alpha subunits and $\mathrm{p} 21$ (RAS). J Biol Chem 273(25):15830-15837

El-Husseini AE, Bredt DS (2002) Protein palmitoylation: a regulator of neuronal development and function. Nat Rev Neurosci 3(10): 791-802

El-Husseini AE, Craven SE, Chetkovich DM et al (2000) Dual palmitoylation of PSD-95 mediates its vesiculotubular sorting, postsynaptic targeting, and ion channel clustering. J Cell Biol 148(1): $159-172$

El-Husseini AE, Schnell E, Dakoji S et al (2002) Synaptic strength regulated by palmitate cycling on PSD-95. Cell 108(6):849-863

Fukata Y, Fukata M (2010) Protein palmitoylation in neuronal development and synaptic plasticity. Nat Rev Neurosci 11(3):161-175

Fukata M, Fukata Y, Adesnik H, Nicoll RA, Bredt DS (2004) Identification of PSD-95 palmitoylating enzymes. Neuron 44(6): 987-996

Galli LM, Barnes TL, Secrest SS, Kadowaki T, Burrus LW (2007) Porcupine-mediated lipid-modification regulates the activity and distribution of Wnt proteins in the chick neural tube. Development 134(18):3339-3348

Gauthier-Campbell C, Bredt DS, Murphy TH AEE-H (2004) Regulation of dendritic branching and filopodia formation in hippocampal neurons by specific acylated protein motifs. Mol Biol Cell 15(5): 2205-2217

Glaser B, Schumacher J, Williams HJ et al (2005) No association between the putative functional ZDHHC8 single nucleotide polymorphism rs175174 and schizophrenia in large European samples. Biol Psychiatry 58(1):78-80

Glaser B, Moskvina V, Kirov G et al (2006) Analysis of ProDH, COMT and ZDHHC8 risk variants does not support individual or interactive effects on schizophrenia susceptibility. Schizophr Res 87(1-3):21-27

Greaves J, Chamberlain LH (2011) DHHC palmitoyl transferases: substrate interactions and (patho) physiology. Trends Biochem Sci 36(5):245-253

Greaves J, Prescott GR, Gorleku OA, Chamberlain LH (2009) The fat controller: roles of palmitoylation in intracellular protein trafficking 
and targeting to membrane microdomains (Review). Mol Membr Biol 26(1):67-79

Greaves J, Gorleku OA, Salaun C, Chamberlain LH (2010) Palmitoylation of the SNAP25 protein family: specificity and regulation by DHHC palmitoyl transferases. J Biol Chem 285(32): 24629-24638

Gupta P, Soyombo AA, Shelton JM et al (2003) Disruption of PPT2 in mice causes an unusual lysosomal storage disorder with neurovisceral features. Proc Natl Acad Sci U S A 100(21): $12325-12330$

Haltia M (2006) The neuronal ceroid-lipofuscinoses: from past to present. Bba-Mol Basis Dis 1762(10):850-856

Hellsten E, Vesa J, Olkkonen VM, Jalanko A, Peltonen L (1996) Human palmitoyl protein thioesterase: evidence for lysosomal targeting of the enzyme and disturbed cellular routing in infantile neuronal ceroid lipofuscinosis. The EMBO j 15(19):5240-5245

Hofmann K (2000) A superfamily of membrane-bound Oacyltransferases with implications for wnt signaling. Trends Biochem Sci 25(3):111-112

Huang K, El-Husseini AE (2005) Modulation of neuronal protein trafficking and function by palmitoylation. Curr Opin Neurobiol 15(5): $527-535$

Huang K, Yanai A, Kang R et al (2004) Huntingtin-interacting protein HIP14 is a palmitoyl transferase involved in palmitoylation and trafficking of multiple neuronal proteins. Neuron 44(6):977-986

Huang K, Sanders S, Singaraja R et al (2009) Neuronal palmitoyl acyl transferases exhibit distinct substrate specificity. FASEB $\mathrm{j}:$ off pub of the Fed of Am Soc for Exp Biol 23(8):2605-2615

Kalchman MA, Graham RK, Xia G et al (1996) Huntingtin is ubiquitinated and interacts with a specific ubiquitin-conjugating enzyme. J Biol Chem 271(32): 19385-19394

Kang R, Wan J, Arstikaitis P et al (2008) Neural palmitoylproteomics reveals dynamic synaptic palmitoylation. Nature 456(7224):904-909

Kato H, Allen ND, Emson PC, Kiyama H (2000) GAP-43 N-terminal translocation signal targets beta-galactosidase to developing axons in a pan-neuronal transgenic mouse line. Brain Res Dev Brain Res 121(1):109-112

Kim E, Sheng M (2004) PDZ domain proteins of synapses. Nat Rev Neurosci 5(10):771-781

Kutzleb C, Sanders G, Yamamoto R et al (1998) Paralemmin, a prenylpalmitoyl-anchored phosphoprotein abundant in neurons and implicated in plasma membrane dynamics and cell process formation. J Cell Biol 143(3):795-813

Laux T, Fukami K, Thelen M, Golub T, Frey D, Caroni P (2000) GAP43, MARCKS, and CAP23 modulate PI $(4,5)$ P (2) at plasmalemmal rafts, and regulate cell cortex actin dynamics through a common mechanism. J Cell Biol 149(7):1455-1472

Levental I, Grzybek M, Simons K (2010) Greasing their way: lipid modifications determine protein association with membrane rafts. Biochemistry 49(30):6305-6316

Levin SW, Baker EH, Zein WM et al (2014) Oral cysteamine bitartrate and $\mathrm{N}$-acetylcysteine for patients with infantile neuronal ceroid lipofuscinosis: a pilot study. Lancet Neurol doi:10.1016/S14744422(14)70142-5

Linder ME, Deschenes RJ (2007) Palmitoylation: policing protein stability and traffic. Nat Rev Mol Cell Biol 8(1):74-84

Liu H, Abecasis GR, Heath SC et al (2002) Genetic variation in the 22q11 locus and susceptibility to schizophrenia. Proc Natl Acad Sci U S A 99(26):16859-16864

Lobo S, Greentree WK, Linder ME, Deschenes RJ (2002) Identification of a Ras palmitoyltransferase in Saccharomyces cerevisiae. J Biol Chem 277(43):41268-41273

Lyly A, Marjavaara SK, Kyttala A et al (2008) Deficiency of the INCL protein Ppt1 results in changes in ectopic F1-ATP synthase and altered cholesterol metabolism. Hum Mol Genet 17(10):1406-1417
Mansilla F, Birkenkamp-Demtroder K, Kruhoffer M et al (2007) Differential expression of DHHC9 in microsatellite stable and instable human colorectal cancer subgroups. Br J Cancer 96(12): 1896-1903

Mansouri MR, Marklund L, Gustavsson P et al (2005) Loss of ZDHHC15 expression in a woman with a balanced translocation $\mathrm{t}(\mathrm{X} ; 15)(\mathrm{q} 13.3 ; \mathrm{cen})$ and severe mental retardation. EJHG 13(8): 970-977

Mill P, Lee AW, Fukata Y et al (2009) Palmitoylation regulates epidermal homeostasis and hair follicle differentiation. PLoS Genet 5(11): e1000748

Mitchell DA, Vasudevan A, Linder ME, Deschenes RJ (2006) Protein palmitoylation by a family of DHHC protein S-acyltransferases. J Lipid Res 47(6):1118-1127

Mitchison HM, Hofmann SL, Becerra CH et al (1998) Mutations in the palmitoyl-protein thioesterase gene (PPT; CLN1) causing juvenile neuronal ceroid lipofuscinosis with granular osmiophilic deposits. Hum Mol Genet 7(2):291-297

Mizumaru C, Saito Y, Ishikawa T et al (2009) Suppression of APP-containing vesicle trafficking and production of betaamyloid by AID/DHHC-12 protein. J Neurochem 111(5): 1213-1224

Mukai J, Liu H, Burt RA et al (2004) Evidence that the gene encoding ZDHHC8 contributes to the risk of schizophrenia. Nat Genet 36(7): $725-731$

Mukai J, Dhilla A, Drew LJ et al (2008) Palmitoylation-dependent neurodevelopmental deficits in a mouse model of $22 \mathrm{q} 11$ microdeletion. Nat Neurosci 11(11):1302-1310

Nadolski MJ, Linder ME (2007) Protein lipidation. FEBS J 274(20): 5202-5210

Nasir J, Floresco SB, O'Kusky JR et al (1995) Targeted disruption of the Huntington's disease gene results in embryonic lethality and behavioral and morphological changes in heterozygotes. Cell 81(5): $811-823$

Ohno Y, Kihara A, Sano T, Igarashi Y (2006) Intracellular localization and tissue-specific distribution of human and yeast DHHC cysteinerich domain-containing proteins. Biochim Biophys Acta 1761(4): 474-483

Otani K, Ujike H, Tanaka Y et al (2005) The ZDHHC8 gene did not associate with bipolar disorder or schizophrenia. Neurosci Lett 390(3): 166-170

Oyama T, Miyoshi Y, Koyama K et al (2000) Isolation of a novel gene on 8p21.3-22 whose expression is reduced significantly in human colorectal cancers with liver metastasis. Genes Chromosomes Cancer 29(1):9-15

Polakis P (2007) The many ways of Wnt in cancer. Curr Opin Genet Dev 17(1):45-51

Politis EG, Roth AF, Davis NG (2005) Transmembrane topology of the protein palmitoyl transferase Akr1. J Biol Chem 280(11):1015610163

Prescott GR, Gorleku OA, Greaves J, Chamberlain LH (2009) Palmitoylation of the synaptic vesicle fusion machinery. J Neurochem 110(4):1135-1149

Putilina T, Wong P, Gentleman S (1999) The DHHC domain: a new highly conserved cysteine-rich motif. Mol Cell Biochem 195(1-2): 219-226

Raymond FL, Tarpey PS, Edkins S et al (2007) Mutations in ZDHHC9, which encodes a palmitoyltransferase of NRAS and HRAS, cause $\mathrm{X}$-linked mental retardation associated with a Marfanoid habitus. Am J Hum Genet 80(5):982-987

Resh MD (2006) Palmitoylation of ligands, receptors, and intracellular signaling molecules. Science's STKE : signal transduction knowledge environment 2006 (359): re14

Rocks O, Peyker A, Bastiaens PI (2006) Spatio-temporal segregation of Ras signals: one ship, three anchors, many harbors. Curr Opin Cell Biol 18(4):351-357 
Rocks O, Gerauer M, Vartak N et al (2010) The palmitoylation machinery is a spatially organizing system for peripheral membrane proteins. Cell 141(3):458-471

Roos RA (2010) Huntington's disease: a clinical review. Orphanet j of rare dis 5(1):40

Roth AF, Feng Y, Chen L, Davis NG (2002) The yeast DHHC cysteinerich domain protein Akrlp is a palmitoyl transferase. J Cell Biol 159(1):23-28

Rusch M, Zimmermann TJ, Burger M et al (2011) Identification of acyl protein thioesterases 1 and 2 as the cellular targets of the Rassignaling modulators palmostatin B and $\mathrm{M}$. Angew Chem Int Ed Engl 50(42):9838-9842

Saito S, Ikeda M, Iwata N et al (2005) No association was found between a functional SNP in ZDHHC8 and schizophrenia in a Japanese casecontrol population. Neurosci Lett 374(1):21-24

Salaun C, Greaves J, Chamberlain LH (2010) The intracellular dynamic of protein palmitoylation. J Cell Biol 191(7):1229-1238

Saleem AN, Chen YH, Baek HJ et al (2010) Mice with alopecia, osteoporosis, and systemic amyloidosis due to mutation in Zdhhc13, a gene coding for palmitoyl acyltransferase. PLoS Genet 6(6): e1000985

Santavuori P (2011) The Neuronal ceroid lipofuscinoses, Mole, Sara/ Williams, Ruth/ Goebel, Hans Oxford University Press ELT

Schlesinger MJ, Magee AI, Schmidt MF (1980) Fatty acid acylation of proteins in cultured cells. J Biol Chem 255(21):10021-10024

Schmidt MF, Schlesinger MJ (1979) Fatty acid binding to vesicular stomatitis virus glycoprotein: a new type of post-translational modification of the viral glycoprotein. Cell 17(4):813-819

Shin HD, Park BL, Bae JS et al (2010) Association of ZDHHC8 polymorphisms with smooth pursuit eye movement abnormality. Am j of med genet Part B, Neuropsychiatr genet : the official publication of the Int Soc of Psychiatr Genet 153B(6):1167-1172

Shindou H, Shimizu T (2009) Acyl-CoA:lysophospholipid acyltransferases. J Biol Chem 284(1):1-5

Singaraja RR, Hadano S, Metzler M et al (2002) HIP14, a novel ankyrin domain-containing protein, links huntingtin to intracellular trafficking and endocytosis. Hum Mol Genet 11(23):2815-2828

Singaraja RR, Huang K, Sanders SS et al (2011) Altered palmitoylation and neuropathological deficits in mice lacking HIP14. Hum Mol Genet 20(20):3899-3909

Slow EJ, van Raamsdonk J, Rogers D et al (2003) Selective striatal neuronal loss in a YAC128 mouse model of Huntington disease. Hum Mol Genet 12(13):1555-1567

Soyombo AA, Hofmann SL (1997) Molecular cloning and expression of palmitoyl-protein thioesterase 2 (PPT2), a homolog of lysosomal palmitoyl-protein thioesterase with a distinct substrate specificity. J Biol Chem 272(43):27456-27463

Sturrock A, Leavitt BR (2010) The clinical and genetic features of Huntington disease. J Geriatr Psychiatry Neurol 23(4):243-259

Takada R, Satomi Y, Kurata T et al (2006) Monounsaturated fatty acid modification of Wnt protein: its role in Wnt secretion. Dev Cell 11(6):791-801

Tian L, McClafferty H, Jeffries O, Shipston MJ (2010) Multiple palmitoyltransferases are required for palmitoylation-dependent regulation of large conductance calcium- and voltage-activated potassium channels. J Biol Chem 285(31):23954-23962

Tomatis VM, Trenchi A, Gomez GA, Daniotti JL (2010) Acyl-protein thioesterase 2 catalyzes the deacylation of peripheral membraneassociated GAP-43. PLoS ONE 5(11):e15045

Tyynela J, Palmer DN, Baumann M, Haltia M (1993) Storage of saposins $\mathrm{A}$ and $\mathrm{D}$ in infantile neuronal ceroid-lipofuscinosis. FEBS Lett 330(1):8-12

Veit M, Serebryakova MV, Kordyukova LV (2013) Palmitoylation of influenza virus proteins. Biochem Soc Trans 41(1):50-55

Verkruyse LA, Hofmann SL (1996) Lysosomal targeting of palmitoylprotein thioesterase. J Biol Chem 271(26):15831-15836

Vesa J, Hellsten E, Verkruyse LA et al (1995) Mutations in the palmitoyl protein thioesterase gene causing infantile neuronal ceroid lipofuscinosis. Nature 376(6541):584-587

Vetrivel KS, Meckler X, Chen Y et al (2009) Alzheimer disease Abeta production in the absence of S-palmitoylationdependent targeting of BACE1 to lipid rafts. J Biol Chem 284(6):3793-3803

Wang X, Reid Sutton V, Omar Peraza-Llanes J et al (2007) Mutations in $\mathrm{X}$-linked PORCN, a putative regulator of Wnt signaling, cause focal dermal hypoplasia. Nat Genet 39(7):836-838

Warby SC, Visscher H, Collins JA et al (2011) HTT haplotypes contribute to differences in Huntington disease prevalence between Europe and East Asia. EJHG 19(5):561-566

Willert K, Brown JD, Danenberg E et al (2003) Wnt proteins are lipidmodified and can act as stem cell growth factors. Nature 423(6938): $448-452$

Xu M, St Clair D, He L (2010) Testing for genetic association between the ZDHHC8 gene locus and susceptibility to schizophrenia: an integrated analysis of multiple datasets. Am j of med genet Part B, Neuropsychiatr genet : the official publication of the Int Soc of Psychiatr Genet 153B(7):1266-1275

Yamamoto Y, Chochi Y, Matsuyama H et al (2007) Gain of 5p15.33 is associated with progression of bladder cancer. Oncology 72(1-2): $132-138$

Yanai A, Huang K, Kang R et al (2006) Palmitoylation of huntingtin by HIP14 is essential for its trafficking and function. Nat Neurosci 9(6): 824-831

Yeh DC, Duncan JA, Yamashita S, Michel T (1999) Depalmitoylation of endothelial nitric-oxide synthase by acyl-protein thioesterase 1 is potentiated by $\mathrm{Ca}(2+)$-calmodulin. J Biol Chem 274(46): 33148-33154

Zeidman R, Jackson CS, Magee AI (2009) Protein acyl thioesterases (Review). Mol Membr Biol 26(1):32-41

Zhang Z, Lee YC, Kim SJ et al (2006) Palmitoyl-protein thioesterase1 deficiency mediates the activation of the unfolded protein response and neuronal apoptosis in INCL. Hum Mol Genet 15(2):337-346

Zhang J, Planey SL, Ceballos C, Stevens SM Jr, Keay SK, Zacharias DA (2008) Identification of CKAP4/p63 as a major substrate of the palmitoyl acyltransferase DHHC2, a putative tumor suppressor, using a novel proteomics method. Mol \& cel proteomics : MCP 7(7):1378-1388 